\title{
Comparative Study in Performance for Subcarrier Mapping in Uplink 4G-LTE under Different Channel Cases
}

\author{
Raad Farhood Chisab ${ }^{1,2}$ \\ ${ }^{1}$ Foundation of Technical Education, IRAQ \\ ${ }^{2}$ Dept. of ECE, SHIATS (Deemed to be University) \\ Allahabad-211 007, UP, INDIA
}

\author{
Prof. (Dr.) C. K. Shukla \\ Prof. at Dept. of ECE, SHIATS, \\ (Deemed to be University) Allahabad-211 007, \\ UP, INDIA
}

\begin{abstract}
-in recent years, wireless communication has experienced a rapid growth and it promises to become a globally important infrastructure. One common design approach in fourth generation 4G systems is Single Carrier Frequency Division Multiple Access (SC-FDMA). It is a single carrier communication technique on the air interface. It has become broadly accepted mainly because of its high resistance to frequency selective fading channels. The third Generation Partnership Project-Long Term Evolution (3GPP-LTE) uses this technique in uplink direction because of its lower peak to average power ratio PAPR as compared to Orthogonal Frequency Division Multiple Access (OFDMA) that is used for downlink direction. In this paper the LTE in general and SCFDMA will be discuss in details and its performance will be study under two types of subcarrier mapping which are localized and distributed mode also within different channel cases. The results show that the localized subcarrier mapping give lower bit error rate BER than the distributed mode and give different activity under miscellaneous channel cases.
\end{abstract}

\section{Keywords-LTE; SCFDMA; 4G; PAPR; BER; channel model}

\section{INTRODUCTION}

Wireless communications is an emerging field which has seen enormous growth in the last several years. The unprecedented and ubiquitous use of mobile phone technology, rapid expansion in wireless local area networks (WLAN) and the exponential growth of the Internet have resulted in an increased demand for new methods of establishing high capacity wireless networks. As the wireless standards evolved, the access techniques used also exhibited increase in efficiency, capacity and scalability. The first generation wireless standards used Frequency Division Multiple Access (FDMA) or Time Division Multiple Access (TDMA) [1]. In wireless channels, FDMA consumed more bandwidth for guard to avoid intercarrier interference (ICI) and TDMA proved to be less efficient in handling high data rate channels as it requires large guard periods to alleviate the multipath impact. 4G (4th Generation) mobile networks are evolving to provide a comprehensive IPbased integrated solution at an affordable price where voice, data and streamed multimedia can be given to users on an anytime, anywhere basis, and at higher data rates than previous generations. This will be achieved after the convergence of all types of wired and wireless technologies and will be capable of providing data rates between $100 \mathrm{Mbps}$ and 1Gbps (both indoors and outdoors), with premium quality and high security.
High data rate calls upon an improved spectral efficiency. The Third Generation Partnership Project Long Term Evolution (3GPP-LTE) has been standardized for the emerging 4th generation (4G) wireless communications [2]. The OFDMA and SCFDMA are technique the most prominent candidates that are used in $4 \mathrm{G}$ mobile systems. The LTE decided to use the OFDMA for downlink and using the SCFDMA for uplink [3]. The choice of SCFDMA in uplink direction comes as a result of its ability to reduce the PAPR as compare with the OFDMA and also give lower BER in case of localized mode than the distributed mode.

\section{LONG TERM EVOLUTION (LTE)}

LTE system is expected to be competitive for many years to come, therefore, the requirements and targets set forth for this system are quite stringent. The main objectives of the evolution are to further improve service provisioning and reduce user/operator costs. The parameter of LTE can be summarized in table 1 [4]. A key requirement for LTE is to make possible a seamless transition from current telecommunication systems. This can be made possible by reuse of the current spectrums, interoperability between current and upcoming system, reuse of existing sites and production competitively priced equipment. It gives the operators the ability to migrate to new systems with ease [5]. But this requires adoption of simplified system architecture, stringent limits on spectrum and usage of a new radio-access technology with better characteristics. Transmission parameters in LTE consist of frequency, space, and time to create transmission resources for carrying data [6].

All the LTE signals derive their timing from a clock operating at $30.72 \mathrm{MHz}=15 \mathrm{kHz} \times 2048$. This is the timing required for the 2048 point discrete Fourier transform (DFT) specified for $20 \mathrm{MHz}$ channels. Therefore, the basic time interval in an LTE physical channel is one clock period of duration $T_{s}=1 /(15 k \times 2048)=32.255 n s$ per clock period. The LTE radio frame for downlink and uplink transmission is $307200 \times T_{S}=10 \mathrm{~ms}$ long. LTE supports two radio frame structures which are frequency division duplex FDD which uses type 1 frame structure and time division duplex TDD which is applicable to type 2 frame structure [7].

A radio frame consists of 10 sub frames $30720 \times T_{S}=$ $1 m s$ in FDD and two half- frames $153600 \times T_{S}=5 \mathrm{~ms}$ in 
TDD. A half-frame is divided into four subframes and a special subframe, or five subframes, based on downlink to uplink switch point periodicity. The TDD frame structure can be configured in seven different sub frame formats. The sub frames 0 and 5 and DwPTS (downlink pilot timing slot) are reserved for downlink transmission. The sub frame that appears after special sub frame as well as UpPTS (uplink pilot timing slot), is always assigned to uplink transmission. Each sub frame in both FDD and TDD has two slots of $15360 \times T_{S}=0.5 \mathrm{~ms}$.

TABLE I. THE IMPORTANT PARAMETERS FOR LTE-SCFDMA

\begin{tabular}{|c|c|c|c|c|c|c|}
\hline Parameters & \multicolumn{6}{|c|}{ value } \\
\hline BW (MHz) & 1.25 & 2.5 & 5 & 10 & 15 & 20 \\
\hline Resource Block & 6 & 12 & 25 & 50 & 75 & 100 \\
\hline FFT Size & 128 & 256 & 512 & 1024 & 1536 & 2048 \\
\hline$f_{\mathrm{s}}(\mathrm{MHz})$ & 1.92 & 3.84 & 7.68 & 15.36 & 23.04 & 30.72 \\
\hline Sample per slot & 960 & 1920 & 3840 & 7680 & 11520 & 15360 \\
\hline No. of sub carrier & 76 & 151 & 301 & 601 & 901 & 1201 \\
\hline Carrier spacing & \multicolumn{6}{|c|}{$15 \mathrm{KHz}$} \\
\hline (PRB) BW & \multicolumn{6}{|c|}{$180 \mathrm{KHz}$} \\
\hline $\begin{array}{l}\text { No. of OFDM } \\
\text { symbol/slot }\end{array}$ & \multicolumn{6}{|c|}{7 for normal $\mathrm{CP}$ and 6 for extended $\mathrm{CP}$} \\
\hline Full mobility & \multicolumn{6}{|c|}{ Up to $500 \mathrm{Km} / \mathrm{h}$} \\
\hline Capacity & \multicolumn{6}{|c|}{$>200$ User per cell } \\
\hline Cell size & \multicolumn{6}{|c|}{$5-100 \mathrm{Km}$} \\
\hline
\end{tabular}

A resource element, consisting of one subcarrier during one OFDM symbol, is the smallest physical resource in LTE. Furthermore, as illustrated in Fig. 1, resource elements are grouped into physical resource block (PRB), where each physical resource block consists of a bandwidth equal to 180 $\mathrm{kHz}$ (12 consecutive subcarriers) in the frequency domain and one $0.5 \mathrm{~ms}$ (one slot) in the time domain [8].

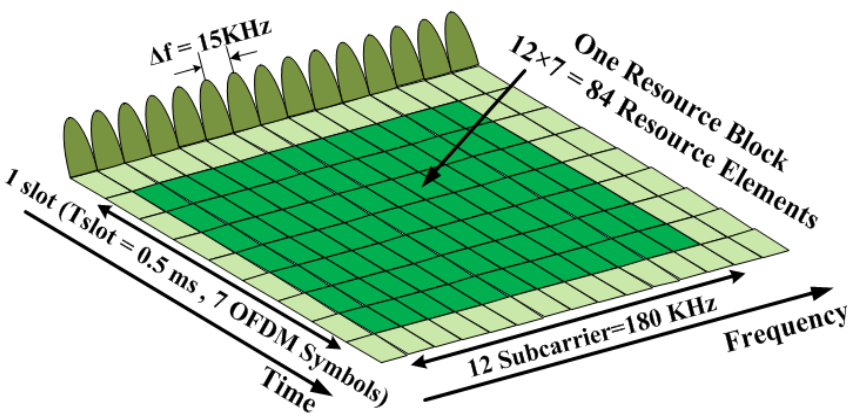

Fig. 1. The resource block and subcarrier in LTE-SCFDMA

Depending on the cyclic prefix (CP) type, which is a copy of the last portion of the data symbol which is inserted in front of the same data symbol during the guard interval, LTE employed two types of cyclic prefix, namely normal $\mathrm{CP}$ and extended CP [9]. The duration of an extended cyclic prefix is 512 clock periods, $512 \times T S=16.67 \mu$ sec. In slots with seven symbols, the duration of a normal cyclic prefix is 160 clock periods, $160 \times T S=5.21 \mu \mathrm{sec}$, for the first symbol and 144 clock periods, $144 \times T S=4.69 \mu \mathrm{sec}$, for the other six symbols [7].

\section{SC-FDMA}

There is considerable interest in the use of Single Carrier Frequency Division Multiple Access (SC-FDMA) as the uplink transmission scheme in the 3GPP-LTE standard. This interest is justified by the inherent single carrier structure of SCFDMA, which results in reduced sensitivity to phase noise and a lower Peak-to-Average Power Ratio compared to Orthogonal Frequency Division Multiple Access OFDMA [9].

SC-FDMA, which utilizes single carrier modulation and frequency domain equalization, is a technique that has similar throughput and essentially the same overall structure as OFDMA [10]. One advantage over OFDMA is that the SCFDMA signal has lower peak-to-average power ratio (PAPR) because of its inherent single carrier structure. SC-FDMA has attracted attention as an alternative to OFDMA especially in uplink communications where lower PAPR benefits the mobile terminal in terms of transmit power efficiency. SC-FDMA has been adopted as the uplink multiple access scheme for the 3rd Generation Partnership Project Long Term Evolution [11].

As shown in Fig. 2, the transmitter of an SC-FDMA system converts a binary input signal to a sequence of modulated subcarriers. At the input to the transmitter, a baseband modulator transforms the binary input to a multilevel sequence of complex numbers $x_{n}$ in one of several possible modulation formats. The transmitter next groups the modulation symbols $\left\{x_{n}\right\}$ into blocks each containing $N$ symbols. The first step in modulating the SC-FDMA subcarriers is to perform an $N$-point DFT to produce a frequency domain representation $X_{k}$ of the input symbols. The DFT equation is represented as [12]:

$$
X_{k}=\sum_{k=0}^{N-1} x_{n} e^{\frac{-2 \pi j k n}{N}} k=0,1 \ldots N-1
$$

It then maps each of the $N$ DFT outputs to one of the $M$ (> $N$ ) orthogonal subcarriers that can be transmitted. If $N=M / Q$ and all terminals transmit $N$ symbols per block, the system can handle $Q$ simultaneous transmissions without co-channel interference. $Q$ is the bandwidth expansion factor of the symbol sequence. The result of the subcarrier mapping is the set $\widetilde{X}_{l}(l=$ $0,1,2 \ldots, M-1)$ of complex subcarrier amplitudes, where $N$ of the amplitudes are non-zero. As in OFDMA, an $M$-point IDFT transforms the subcarrier amplitudes to a complex time domain signal $\tilde{x}_{m}$. The Inverse discrete Fourier transform IDFT equation is represented as [12]:

$$
\tilde{x}_{m}=\frac{1}{M} \sum_{l=0}^{M-1} X_{l} e^{\frac{2 \pi j k m}{M}} m=0,1 \ldots M-1
$$

There are $M$ subcarriers, among which $N(<M)$ subcarriers are occupied by the input data. In the time domain, the input data symbol has symbol duration of $T$ seconds and the symbol duration is compressed to $\widetilde{T}=T \frac{N}{M}$ seconds after going through SC-FDMA modulation.

There are two types of sub-carrier mapping which are localized and distributed mapping as shown in Fig. 3. In localized mapping the output from the DFT is mapped to a subset of consecutive subcarrier, confining only to a fraction of system bandwidth and the zero padding process is done either at the first or last, but the outputs of the DFT will be placed in the sequence order without any interchanging [13]. In 
distributed mapping the output of the DFT is assigned, noncontinuously to the sub-carrier, over the entire bandwidth and the zero padding is done equally over the entire bandwidth [14]. The data block consists of $\mathrm{N}$ complex modulation symbols generated at a rate $\mathrm{R}_{\text {source }}(\mathrm{symbols} / \mathrm{sec})$. The $\mathrm{N}$-point FFT produces $\mathrm{N}$ frequency-domain symbols that modulate $\mathrm{N}$ out of $\mathrm{M}$ orthogonal sub-carriers spread over a bandwidth $W$. The sub-carriers mapping process can be shown in Fig. 4. Where $W$ can be defined as [15]:

$$
W=M \cdot F_{0} \quad H z
$$

Where $F_{0}(\mathrm{~Hz})$ is the sub-carriers frequency spacing. The channel transmission rate is:

$$
R_{\text {channel }}=[M / N] \cdot R_{\text {source }} \quad(\text { Symbol } / \mathrm{sec})
$$

The bandwidth spreading factor $\mathrm{Q}$ is given by:

$$
Q=R_{\text {channel }} / R_{\text {source }}=M / N
$$

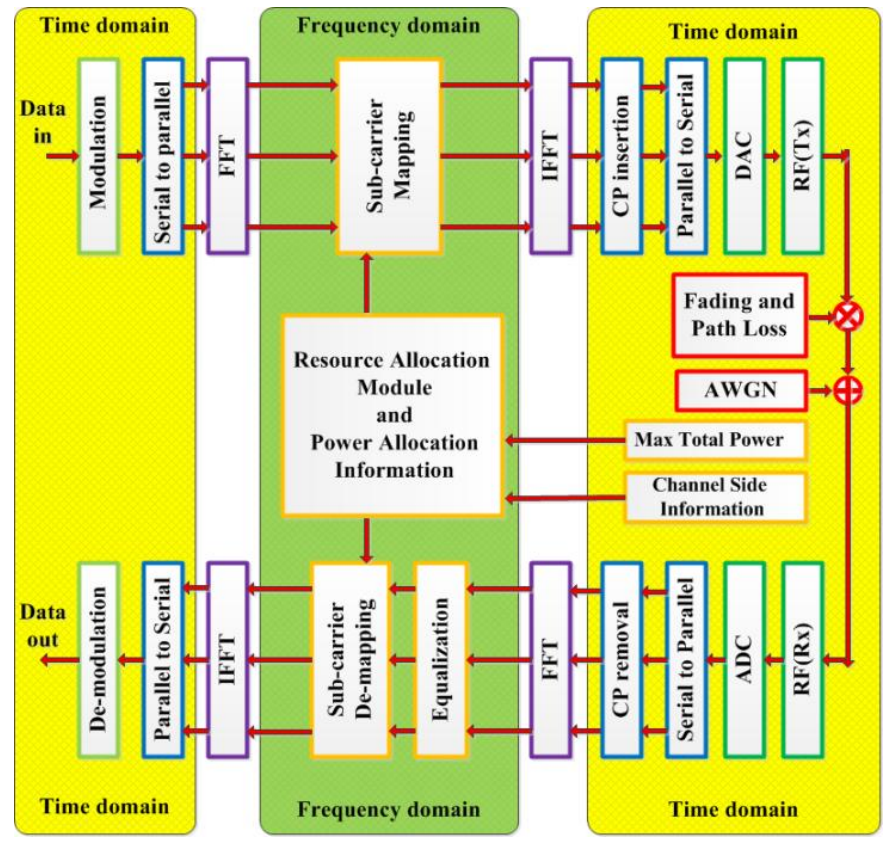

Fig. 2. The block diagram of the SCFDMA system

For LFDMA, the frequency samples after subcarrier mapping $\left\{\tilde{X}_{l}\right\}$ can be described as follows [13]:

$$
\tilde{X}_{l}=\left\{\begin{array}{l}
X_{l}, 0 \leq l \leq N-1 \\
0,0 \leq l \leq M-1
\end{array}\right.
$$

Let $m=Q \cdot n+q$, where $0 \leq n \leq N-1$

$$
\text { and } 0 \leq q \leq Q-1 \text { Then }
$$

$$
\begin{aligned}
& \tilde{x}_{m}=\tilde{x}_{Q n+q}=\frac{1}{M} \sum_{l=0}^{M-1} x_{l} e^{j 2 \pi i \frac{m}{M}} \\
& =\frac{1}{Q} \frac{1}{N} \sum_{l=0}^{n} x_{l} e^{j 2 \pi i \frac{Q n+q}{Q N}} x_{l}
\end{aligned}
$$

If $\mathrm{q}=0$ then

$$
\tilde{x}_{m}=\tilde{x}_{Q n}=\frac{1}{Q} \frac{1}{N} \sum_{l=0}^{N-1} X_{l} e^{j 2 \pi l \frac{Q n}{Q N}}
$$

$$
\begin{aligned}
& =\frac{1}{Q} \frac{1}{N} \sum_{l=0}^{N-1} X_{l} e^{j 2 \pi l \frac{n}{N}} \\
& =\frac{1}{Q} x_{n}=\frac{1}{Q} x_{(m)_{\bmod N}}
\end{aligned}
$$

If $q \neq 0$, since $X_{l}=\sum_{p=0}^{N-1} x_{p} e^{-j 2 \pi l \frac{p}{N}}$ then eqn. 6 can be expressed as follows:

$$
\begin{aligned}
& \tilde{x}_{m}=x_{Q n+q}=\frac{1}{Q} \frac{1}{N} \sum_{l=0}^{N-1} X_{l} e^{j 2 \pi l \frac{Q n+q}{Q N}} \\
& =\frac{1}{Q} \frac{1}{N} \sum_{l=0}^{N-1}\left(\sum_{p=0}^{N-1} x_{p} e^{-j 2 \pi l \frac{p}{N}}\right) e^{J 2 \pi l \frac{Q n+q}{Q N}} \\
& =\frac{1}{Q} \frac{1}{N} \sum_{l=0}^{N-1} \sum_{p=0}^{N-1} x_{p} e^{j 2 \pi l\left\{\frac{(n-p)}{N}+\frac{q}{Q N}\right\}} \\
& =\frac{1}{Q} \frac{1}{N} \sum_{p=0}^{N-1} x_{p}\left(\sum_{i=0}^{n} e^{j 2 \pi l\left\{\frac{(n-p)}{N}+\frac{q}{Q N}\right\}}\right) \\
& =\frac{1}{Q} \frac{1}{N} \sum_{p=0}^{N-1} x_{p} \frac{1-e^{j 2 \pi(n-p)} e^{j 2 \pi \frac{q}{Q}}}{1-e^{J 2 \pi\left\{\frac{(N-P)}{N}+\frac{q}{Q N}\right\}}} \\
& =\frac{1}{Q} \frac{1}{N} \sum_{p=0}^{N-1} x_{p} \frac{1-e^{j 2 \pi \frac{q}{Q}}}{1-e^{J 2 \pi\left\{\frac{(n-p}{N}+\frac{q}{Q N}\right\}}} \\
& =\frac{1}{Q}\left(1-e^{\left.J 2 \pi \frac{q}{Q}\right)} \frac{1}{N} \sum_{p=0}^{N-1} \frac{x_{p}}{1-e^{j 2 \pi\left\{\frac{(n-p)}{N}+\frac{q}{Q N}\right\}}}\right.
\end{aligned}
$$

As can be seen from eqn. 9 and 16, LFDMA signal in the time domain has exact copies of input time symbols with a scaling factor of 1/Q in the N-multiple sample positions and in between values are sum of all the time input symbols in the input block with different complex-weighting.

Now, For DFDMA, the frequency samples after subcarrier mapping $\tilde{X}_{l}$ can be described as follows.

$$
\tilde{X}_{l}=\left\{\begin{array}{l}
X_{l / \tilde{Q}}, l=\tilde{Q} \cdot k(0 \leq k \leq N-1) \\
0 \quad, \text { otherwise }
\end{array}\right.
$$

Where $0 \leq l \leq M-1, M=Q \cdot N$, and $1 \leq \tilde{Q} \leq Q$

Let $m=Q \cdot n+q(0 \leq n \leq N-1,0 \leq q \leq Q-1)$

Then

$$
\begin{aligned}
& \tilde{x}_{m}\left(=\tilde{x}_{Q . n+q}\right)=\frac{1}{M} \sum_{l=0}^{N-1} \tilde{X}_{l} e^{j 2 \pi l \frac{m}{M}} \\
& =\frac{1}{Q} \frac{1}{N} \sum_{k=0}^{N-1} X_{k} e^{j 2 \pi \tilde{Q} k \frac{Q n+q}{Q N}}
\end{aligned}
$$

If $\mathrm{q}=0$ then

$$
\begin{gathered}
\tilde{x}_{m}=\tilde{x}_{Q . n}=\frac{1}{Q} \frac{1}{N} \sum_{k=0}^{N-1} X_{k} e^{j 2 \pi \tilde{Q} k \frac{Q n}{Q N}} \\
=\frac{1}{Q} \frac{1}{N} \sum_{i=0}^{n} X_{k} e^{j 2 \pi \tilde{Q} k \frac{n}{N}} \\
=\frac{1}{Q} \frac{1}{N} \sum_{i=0}^{n} X_{k} e^{j 2 \pi k \frac{\widetilde{Q} n}{N}} \\
=\frac{1}{Q}\left(\frac{1}{N} \sum_{i=0}^{n} X_{k} e^{j 2 \pi k \frac{(\widetilde{Q} \cdot n)_{\bmod N}}{N}}\right) \\
\frac{1}{Q} x_{(\tilde{Q} . n)_{\bmod N}}=\frac{1}{Q} x_{\left(Q(m)_{\bmod N}\right)_{\bmod n}}
\end{gathered}
$$


If $q \neq 0$, since $X_{k}=\sum_{p=0}^{N-1} x_{p} e^{-j 2 \pi k \frac{p}{N}} \quad$ Eqn. 21 can be expressed as follows after derivation

$$
\tilde{x}_{m}=\tilde{x}_{Q . n+q}=\frac{1}{Q}\left(1-e^{j 2 \pi q \frac{\widetilde{Q}}{Q}}\right) \frac{1}{N} \sum_{p=0}^{N-1} \frac{x_{p}}{1-e^{j 2 \pi\left\{\frac{(\widetilde{Q} n-p)}{N}+\frac{\tilde{Q} q}{Q N}\right\}}}
$$

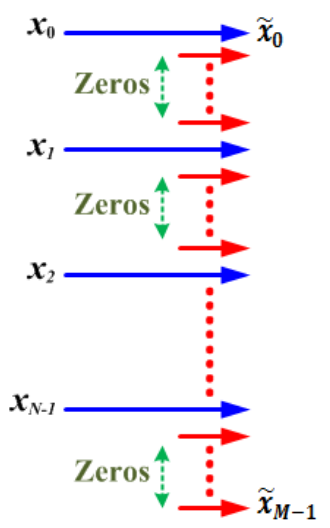

Distributed Mode

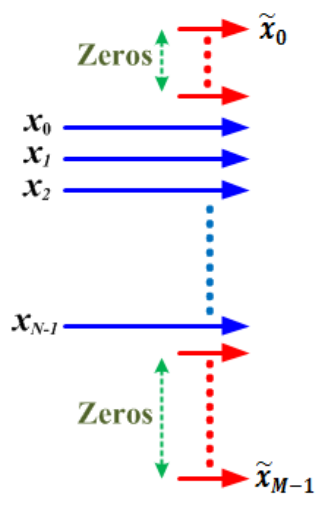

Localized Mode
Fig. 3. The two types of sub-carrier mapping

From a resource allocation point of view, subcarrier mapping methods are further divided into static and channeldependent scheduling (CDS) methods. CDS assigns subcarriers to users according to the channel frequency response of each user [16]. CDS is of great benefit with localized subcarrier mapping because it provides significant multi-user diversity which leads to improved system capacity and performance [17]. For these reasons only LFDMA concept is proposed to use in the 3GPP-LTE specifications.

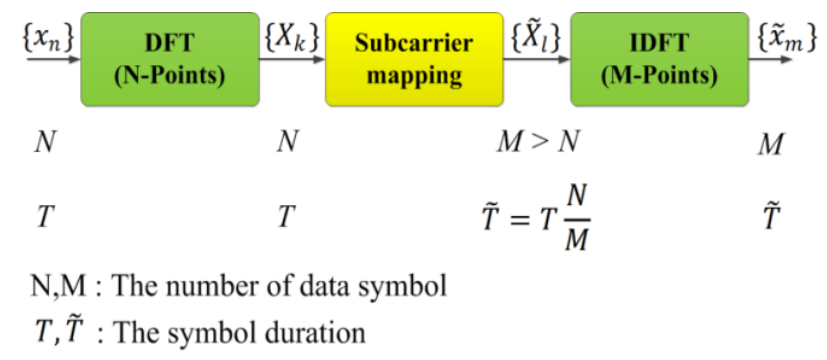

Fig. 4. The process of sub-carriers mapping

The transmitter performs two other signal processing operations prior to transmission. It inserts a set of symbols referred to as a cyclic prefix (CP) in order to provide a guard time to prevent inter-block interference due to multi-path propagation. The transmitter also performs a linear filtering operation referred to as pulse shaping in order to reduce out-ofband signal energy. The receiver transforms the received signal into the frequency domain via DFT, de-maps the subcarriers, and then performs frequency domain equalization (that will be discussed later). The equalized symbols are transformed back to the time domain by means of an IDFT, and detection and decoding take place in the time domain [18]. After the Subcarrier de-mapping is done. The de-mapped signal is given to the IDFT to get the time domain signal back. The IDFT output is given for QPSK or QAM demodulation. After the demodulation the receiver generate the final bit stream [19].

\section{ChanNEL EQUALIZATION}

SCFMA suffers from Inter-symbol Interference (ISI) if a transmission over a frequency selective channel is considered. Therefore for mobile radio applications, SCFDMA requires Equalization at the receiver. The only use of Guard Interval (GI) for equalization does not meet the challenges of the future mobile radio system because of the reduction of spectral efficiency [20].

Channel equalization is one of the key blocks in LTE receiver. It is one of the most important elements of wireless receivers that employ coherent demodulation. For practical LTE systems, it is important to have an equalization technique that is specifically designed for LTE pilots, and has low computational and hardware complexities. An equalizer within a receiver compensates for the average range of the expected channel amplitude and delay characteristic. Equalizer must be adaptive since the channel is generally unknown and time varying [21].

Equalizer is always used in both time and frequency domains in traditional communication system. In the time domain, for traditional FDM system, equalization is indispensable. Because equalizer is used to balance the channel characteristics in the receiver, equalizer produces the opposite characteristics of channel to offset ISI by time varying multipath channel. But equalization is not a satisfactory method for OFDM system [22]. A possible way to reduce the complexity of linear equalization is to carry out the equalization in the frequency domain. The equalization is carried out block-wise with block size $N$. The sampled received signal is first transformed into the frequency domain by means of a size- $N$ DFT. The equalization is then carried out as frequency-domain filtering [23].

The received signal is equalized in the frequency domain. After the equalization block the equalized signal is then transformed back to the time domain using the IFFT. The method of equalization, which is shown in Fig. 5, is done by the following steps:

Let $\mathrm{E}(\mathrm{m})$ where $\left(\mathrm{m}=0,1,2 \ldots \mathrm{N}_{\mathrm{FFT}}-1\right)$ denote the equalizer coefficient for the $\mathrm{m}^{\text {th }}$ sub carrier, the time domain equalized signal $K(n)$ can be expressed as:

$$
k(n)=\frac{1}{N_{F F T}} \sum_{m=0}^{N_{F F T}-1} E(m) G(m) e^{\frac{i 2 \pi m n}{N_{F F T}}}
$$

Where $n=0,1,2, \ldots, N_{F F T}-1$

The equalizer coefficients $\mathrm{E}(\mathrm{m})$ are determined to minimize the mean square error between the equalized signal and the original signal. The equalizer coefficients are computed according to the types of the frequency domain equalization (FDE) in two methods as follow [24]:

$$
\begin{aligned}
& \text { A. The zero forcing (ZF) Equalizer is } \\
& E(m)=1 / H(m) \quad m=0,1,2, \ldots, N_{F F T}-1
\end{aligned}
$$

B. The Minimum Mean Square Error (MMSE) Equalizer is 


$$
E(m)=H^{*}(m) /\left[|H(m)|^{2}+\left(E_{b} / N_{0}\right)^{-1}\right]
$$

Where $*$ denotes the complex conjugate, $H(m)$ is the transfer function of the channel and $E_{b} / N_{0}$ is average energyper-bit to noise power spectral density. Equalization will be used to eliminate the effect of ISI.

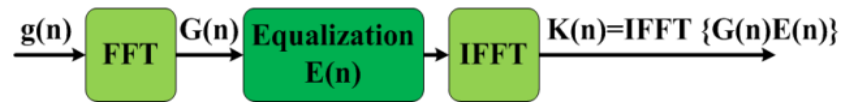

Fig. 5. The process of channel equalization

From Fig. 6 it can be noticed that the MMSE method is better than the ZF method and give lower BER compared with other method. Therefore, in all tests and simulations for channel models, the MMSE method will be use.

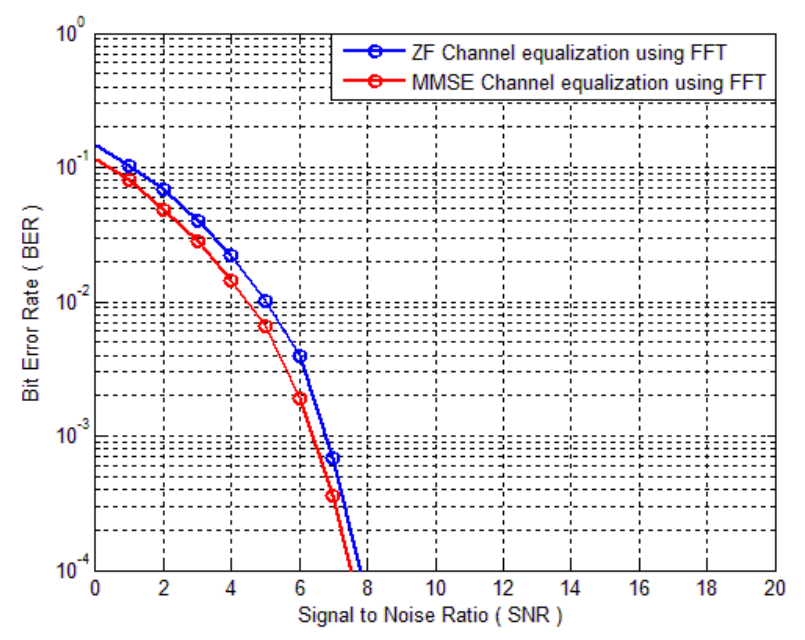

Fig. 6. the performance under two types of channel equalization

In the receiver side, OFDMA utilizes a simple equalizer per subcarrier after FFT. But, SC-FDMA utilizes a complex equalizer before sending the resultant to IFFT. IFFT removes the effect of the FFT in the transmitter. Notice that result of the IFFT is again a time domain signal; the time domain signal is sent to a single detector to create the bits. These differences in receiver side are illustrated in Fig. 7 in which we can see the equalizer simplicity of OFDMA against SC-FDMA. As you can see, SC-FDMA receiver is more complex than OFDMA, but in the transmitter simpler power amplifiers can be utilized to reduce the power consumption. These fortify the SC-FDMA as an uplink transmission scheme, since power efficiency and complexity is important for mobile stations but not in the base station [25].

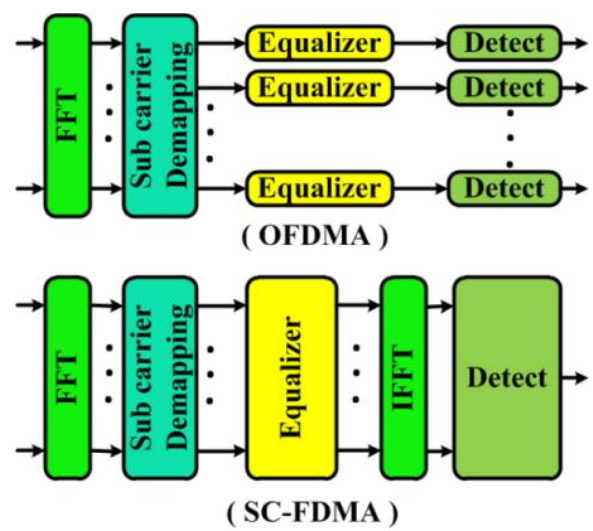

Fig. 7. The equalization in OFDMA and SCFDMA

\section{THE WIRELESS CHANNELS SPECIFICATIONS}

In wire-line communication, the data transmission is primarily corrupted by statistically independent Gaussian noise, as known as the classical additive white Gaussian noise (AWGN). In absence of interference, the primary source of performance degradation in such wire-line channels is thermal noise generated at the receiver. Reliable communication in wireless or radio channels, however, becomes a difficult task as the transmitted data is not only corrupted by AWGN, but also suffers from inter-symbol interference (ISI), in addition to (large-scale and small-scale) fading as well as interference from other users. To master the art of wireless communications, one must understand the propagation characteristics of a radio channel [26]. The fading in radio propagation can be classified into two groups; large-scale fading and small-scale fading as illustrated in Fig. 8. Largescale fading manifests itself as the average signal power attenuation or path loss due to motion over large areas as shown in blocks 1,2 and 3. Small-scale fading refers to the dramatic changes in the signal amplitude and phase that occur due to small changes in the spatial separation between the transmitter and the receiver. As indicated by blocks 4, 5 and 6 in Fig. 8, small-scale fading manifests itself in two mechanisms namely, time-spreading of the signal (or channel dispersion) and time-variant nature of the channel [27]. The signal timespreading (signal dispersion) and time-variant nature of the channel may be examined in two domains, time and frequency, as indicated in block 7, 10, 13 and 16. For signal dispersion nature, we categorize the fading degradation types as frequency selective and frequency non-selective (flat) as illustrated in blocks $8,9,11$, and 12. For time-variant nature, we categorize the fading degradation types as fast fading and slow fading, as shown in blocks $14,15,17$, and 18. 
Large-scale fading is responsible for path-loss in wireless communications and large-scale fading models typically find applications in mobile network planning and understanding free space wireless communication over large areas . In most practical wireless communication systems, the radio communications is far more complex than free-space situation, and is best explained by small-scale fading models [28].

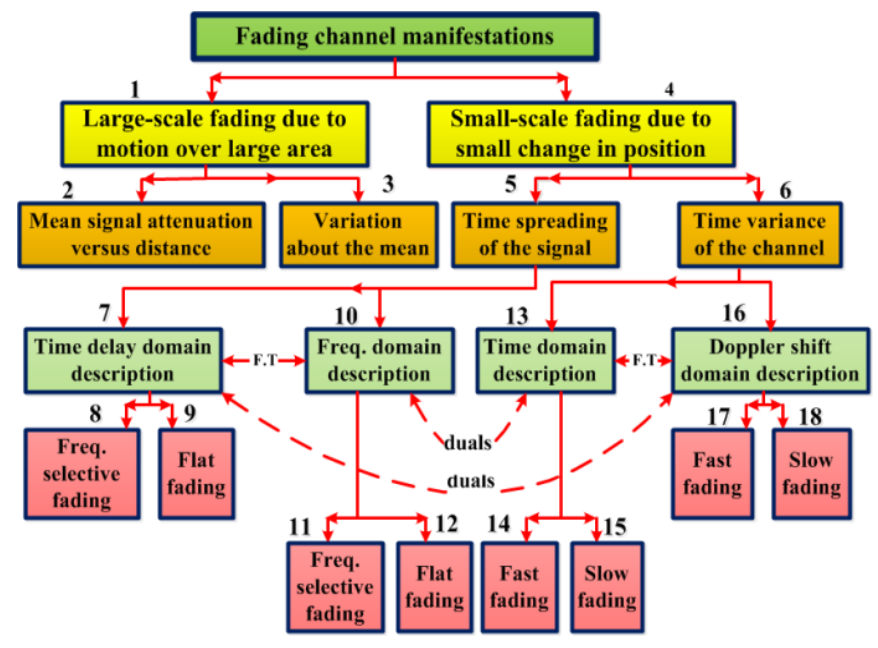

Fig. 8. the fading channel manifestations

Due to the reflection, diffraction and scattering by objects in the environment, transmitted signal propagates through different paths. Thus replicas of the transmitted signal arrive at the receiver with different time delays. This time delay variation is often quantified in terms of delay spread. Larger delay spread means there is a large variation in time delays of different multipath components [29].

The relation between delay spread and OFDM performance can be explained by the principle of frequency diversity. Delay spread is inversely proportional to coherence bandwidth, i.e. larger delay spread results in smaller coherence bandwidth. Coherence bandwidth is the bandwidth over which the channel is considered to be "flat". Within the coherence bandwidth, different signals experience the same channel frequency response. The fading characteristics within coherence bandwidth is flat, thus it is called flat fading. If the bandwidth of a signal is larger than the channel coherence bandwidth, the channel is considered as frequency selective channel. Signals with frequency difference more than coherence bandwidth experience different fading. Therefore, it is called frequency selective fading [30]. The effect of flat and selective fading can be shown in Fig. 9.

An important requirement for assessing technology for Broadband Fixed Wireless Applications is to have an accurate description of the wireless channel model. Channel models are heavily dependent upon the radio architecture. The profile of received signal can be obtained from that of the transmitted signal if we have a model of the medium between the two. This model of the medium is called channel model.
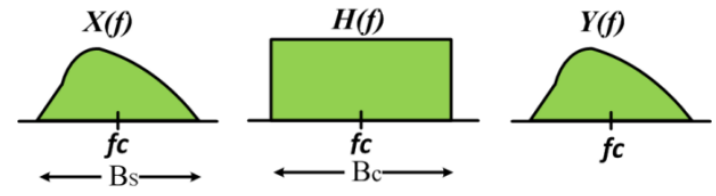

A. Flat fading effect
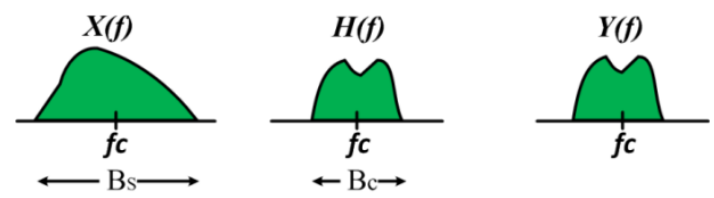

B. Selective fading effect

$$
\begin{aligned}
& \mathbf{B}_{\mathrm{S}}=\text { Bandwidth for signal } \\
& \mathbf{B}_{\mathrm{C}}=\text { Bandwidth for channel }
\end{aligned}
$$

Fig. 9. The effect of flat and selective fading channel on the signal

Channel models are essential tools for simulation and testing of wireless transmission systems. The literature is extensive on this topic, and many standards have recommended channel models for specific propagation environments. These models may characterize path-loss attenuation, shadowing and multipath effects [31]. In this paper some of these channel models will be studies in order to investigate the performance of the system under these channel models. more than one channel model will be discuss and investigate how the system will work under these channel models. These channel models are:

\section{A. COST 207 channel models}

The COST 207 model gives normalized scattering functions, as well as amplitude statistics for four typical environments which are rural area (RA), typical urban area (TU), bad urban area (BU), and hilly terrain (HT). The COST 207 model was presented as an outdoor wireless channel model. This model specifies power gains and time delays for four typical environments [32]. These parameters were evaluated by numerous measurements performed in many countries, including the United Kingdom, France, and Sweden [33]. COST 207 standards provided both the continuous time formula and discrete taps model. The performance of system under these channel models can be shown in Fig. 10 and Fig. 11. Their power distributions are characterized as follows [34]:

For Rural Area (RA):

$$
P(\tau)=\left\{\begin{array}{cc}
\exp (-9.2 \tau) & 0 \leq \tau \leq 0.7 \mu s \\
0 & \text { otherwise }
\end{array}\right\}
$$

For Typical Urban (TU):

$$
P(\tau)=\left\{\begin{array}{cc}
\exp (-\tau) & 0 \leq \tau \leq 7 \mu s \\
0 & \text { otherwise }
\end{array}\right\}
$$

For Bad Urban (BU):

$$
P(\tau)=\left\{\begin{array}{cl}
\exp (-9.2 \tau) & 0 \leq \tau \leq 5 \mu s \\
0.5 \exp (5-\tau) & 5 \leq \tau \leq 10 \mu s \\
0 & \text { otherwise }
\end{array}\right\}
$$

For Hilly Terrain (HT): 


$$
P(\tau)=\left\{\begin{array}{cl}
\exp (-3.5 \tau) & 0 \leq \tau \leq 2 \mu s \\
0.1 \exp (15-\tau) & 15 \leq \tau \leq 20 \mu s \\
0 & \text { otherwise }
\end{array}\right\}
$$

\section{B. COST 259 channel models}

The COST 259 directional channel model (DCM) was developed by the European COST259 project. The COST 259 DCM is wideband and capable of providing channel impulse responses in both spatial and temporal domains. It can also provide these in vertical and horizontal polarization components. It operates at the frequency range from 0.45 to 5 $\mathrm{GHz}$ and bandwidth of less than $10 \mathrm{MHz}$.

The model is very general, and describes the joint effects of small-scale as well as large-scale effects; it covers different cases of macro-cells, micro-cells and Pico-cells. The environments identified so far in COST 259 and typical speeds for each channel type are given in Table II. One of the work items identified in COST 259 is to propose a new set of channel models which overcome the limitations in the GSM channel models, while aiming at the same general acceptance.

The main difference between the COST 259 model and previous models is that it tries to describe the complex range of conditions found in the real world by distributions of channels rather than a few typical cases. The probability densities for the occurrence of different channels are functions of mainly two parameters which are Environment and Distance [35].

TABLE II. Default SPEed For The ChanNel Models

\begin{tabular}{|l|l|}
\hline Channel model & Model speed \\
\hline \multirow{3}{*}{ TUX } & $3 \mathrm{Km} / \mathrm{h}$ \\
\cline { 2 - 2 } & $50 \mathrm{Km} / \mathrm{h}$ \\
\cline { 2 - 2 } & $120 \mathrm{Km} / \mathrm{h}$ \\
\hline \multirow{2}{*}{ RAX } & $120 \mathrm{Km} / \mathrm{h}$ \\
\cline { 2 - 2 } & $250 \mathrm{Km} / \mathrm{h}$ \\
\hline \multirow{2}{*}{ HTX } & $120 \mathrm{Km} / \mathrm{h}$ \\
\hline
\end{tabular}

In the COST 259 model a large number of paths ensure that the correlation properties in the frequency domain are realistic. Path powers follow the exponential channel shapes. There are three types of channel models which are the Rural Area channel model (RAx), The Hilly Terrain channel model (HTx) and the Typical Urban channel model (TUx) [36]. The performance of the system under these channel models can be shown in Fig. 12.

\section{LTE channel models}

The LTE standard adopts models based on the ITU-R M.1225 recommendation and the 3GPP TS 05.05 specification for GSM, widely used in the context of third generation mobile systems [37]. The ITU and 3GPP models are defined by tapped-delay line (TDL) models, where each tap corresponds to a multipath signal characterized by a fixed delay, relative average power and Doppler spectrum. This model use the Pedestrian A and Vehicular A channels from [33], and the Typical Urban (TU) channel from [38], in order to model three reference environments characterized by a low, medium and large delay spread, respectively. Nevertheless, they were designed for a $5 \mathrm{MHz}$ operating bandwidth, and an apparent periodicity appears in their frequency correlation properties for higher bandwidths [39].

The LTE channel models developed by 3GPP are based on the existing 3GPP channel models and ITU channel models. The extended ITU models for LTE were given the name of Extended Pedestrian-A (EPA), Extended Vehicular-A (EVA) and Extended Typical Urban (ETU). These channel models are classified on the basis of low, medium and high delay spread where low delay spreads are used to model indoor environments with small cell sizes while medium and high delay spreads are used to model urban environments with large cells. The high delay spread models are according to Typical Urban GSM model [40]. The performance of the system under these channel models can be shown in Fig. 13.

\section{RESUlTS AND DisCUSSION}

The system (3GPP-LTE-SC-FDMA) based on FFT was simulated and run using MATLAB package version 7.12 (R2011a). The behavior of the proposed system was monitored under the parameters that effect on the performance of the system. These parameters are listed in table III.

The system was tested under three channel cases which are COST207, COST259, and LTE channel models. It can be noticed that the system have different responses under these channel models. The dominant thing that can be seen is that the system under localized subcarrier mapping is better than under distributed mode as shown in Fig. 10 to Fig. 13.

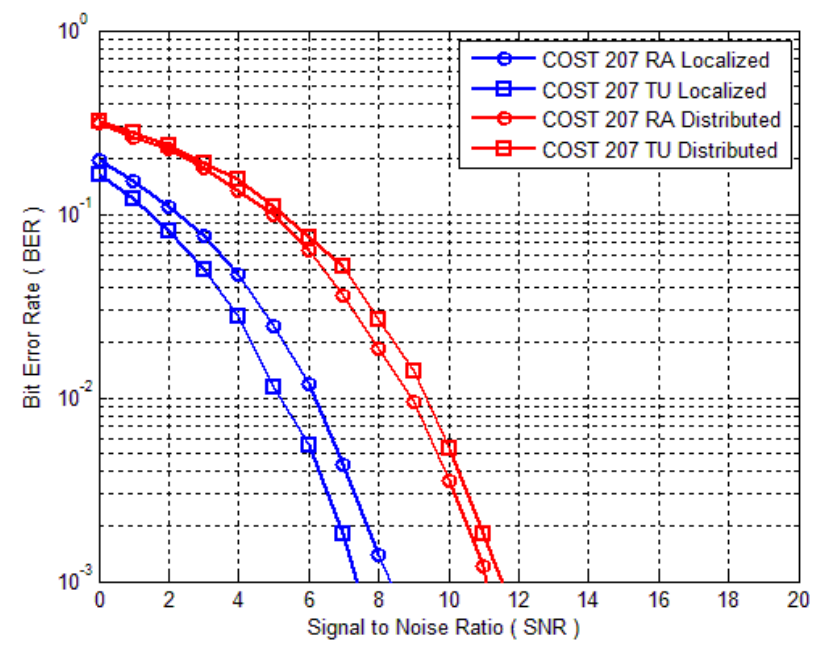

Fig. 10. The performance under COST 207 RA and TU channel models 


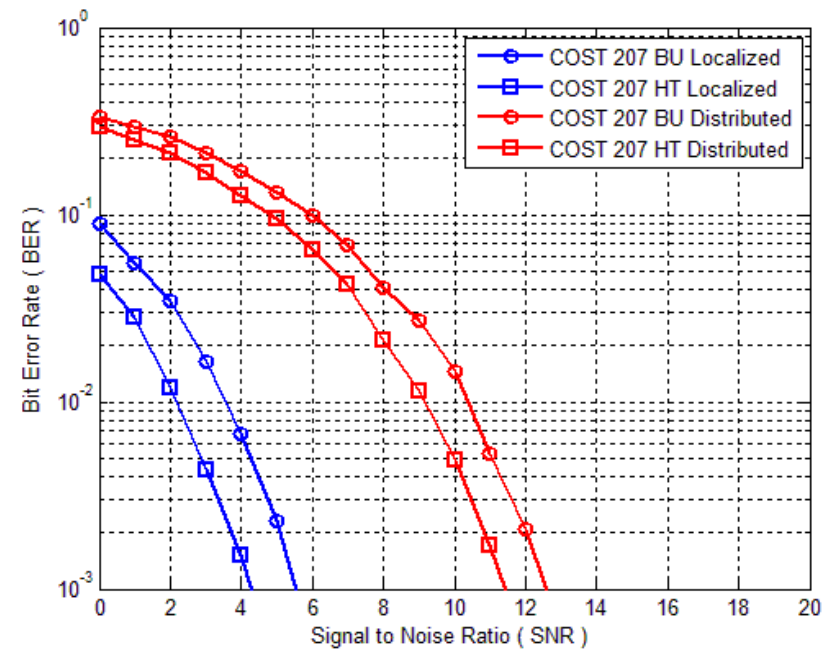

Fig. 11. The performance under COST $207 \mathrm{BU}$ and HT channel models

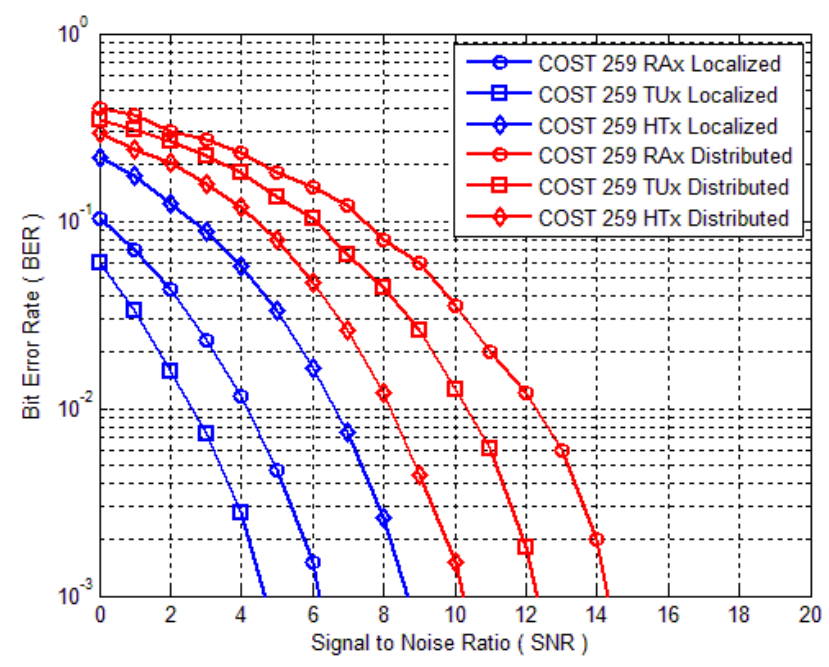

Fig. 12. The performance under COST 259 channel models

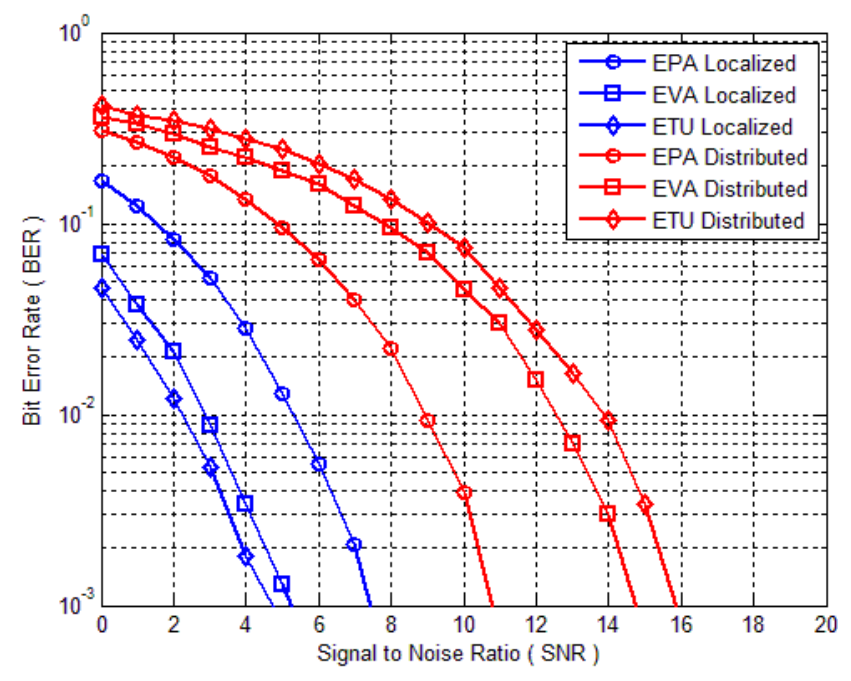

Fig. 13. The performance under LTE channel models
TABLE III. THE PARAMETERS For SiMULATION OF SC-FDMA

\begin{tabular}{|l|l|}
\hline Parameters & Value \\
\hline System bandwidth & $5 \mathrm{MHz}$ \\
\hline Modulation types & QPSK \\
\hline Carrier Frequency ( fc ) & $2025 \mathrm{MHz}$ \\
\hline Sub-carriers spacing & $15 \mathrm{KHz}$ \\
\hline Sub-carriers mapping & Localized, Distributed \\
\hline No. of sub-carrier & 256 \\
\hline Channel equalization & ZF and MMSE \\
\hline Target BER & $10^{-3}$ \\
\hline Channel estimation & Perfect \\
\hline Channel Types & $\begin{array}{l}\text { COST207, COST259, } \\
\text { LTE channels }\end{array}$ \\
\hline
\end{tabular}

VII. CONCLUSION

In this paper the system of SCFDMA was examined under different parameters but the important things is to study the two parameters which are the types of channel equalization and the types of subcarrier mapping. First, when we notice the behavior of system under two type of equalization which are zero forcing (ZF) and minimum mean square error (MMSE), we can notice and monitor the behavior of the system in the Fig. 6 and conclude that the MMSE equalization method was better than the $\mathrm{ZF}$ method and give lower bit error rate (BER) as compare with other method. Second, when notice the behavior of system through the Fig. 10 to Fig. 13, which specify the activity of system when changing the type of subcarrier mapping under different channel cases, it can be noticed that the system is run with better performance under the localized subcarrier mapping method for all types of channel models which are COST207, COST259 and LTE channel models and give lower bit error rate as compare with the other method which depends on the distributed subcarrier mapping. Also we can notice that the system give different activity during the different channel cases because each channel model has its own properties that effects on the system performance.

Finally, from all the results we can conclude that "First: MMSE equalization method is better than the $\mathrm{ZF}$ method, second: the localized subcarrier mapping is better than the distributed mode, third: the activity of the system is changes with different channel models"

\section{ACKNOWLEDGMENT}

In the beginning, I would like to express thanks to my supervisor for giving me the major time and effort and help in order to complete the requirements of doctoral research and come up with good results for knowledge. Also, I would like to extend my thanks, appreciation and pride to the Ministry of Higher Education and Scientific Research in Iraq and particular the Foundation of Technical Education in Baghdad for financial and moral support to scholarship students to complete their studies for reaching to the aim desired.

\section{REFERENCES}

[1] Erik Dahlman, Stefan Parkvall, Johan Sköld and Per Beming, 3G evolution HSPA and LTE for mobile broadband, 2nd ed., Academic Press Elsevier, 2008.

[2] S. Sesia, I. Toufik, and M. Baker, LTE the UMTS Long Term Evolution from theory to practice, 1 st ed.,John Wiley and Sons Ltd., 2009. 
[3] A. Jamalipour, T. Wada, and T. Yamazato, "A Tutorial on multiple access technologies for beyond 3G mobile networks," IEEE Communications Magazine, vol. 43, Issue 2, pp. 110 - 117, February 2005.

[4] 3GPP TS 36.211, Evolved Universal Terrestrial Radio Access (EUTRA); Physical channels and modulation, 2009.

[5] 3GPP TR 25.913, 3GPP:Technical Specification Group Radio Access Network; Requirements for Evolved UTRA and Evolved UTRAN, 2008.

[6] S. Rappaport, Wireless communications: principles and practice, $2 \mathrm{nd}$ ed., Prentice-Hall, 2002.

[7] Ramjee Prasad, OFDM for Wireless communication systems, 2nd ed., Artech house universal Personal communication series, 2008.

[8] D. Astély, E. Dahlman, A. Furuskär, Y. Jading, M. Lindström, and S. Parkvall, "LTE: the evolution of mobile broadband," IEEE Communications Magazine, vol. 47, Issue: 4, pp. 44 - 51, April 2009.

[9] E. Dahlman, H. Ekström, A. Furuskär, Y. Jading, J. Karlsson, M. Lundevall, and S. Parkvall, "The 3G long term evolution - radio interface concepts and performance evaluation," IEEE Vehicular Technology Conference (VTC2006), pp. 137 - 141, May 2006.

[10] Mohamed Noune and Andrew Nix, "A novel frequency-domain implementation of tomlinson-harashima precoding for SC-FDMA," IEEE 69th Vehicular Technology Conference VTC, pp. 1- 5, 2009.

[11] D. Haccoun and G. Begin, "High-rate punctured convolutional codes for viterbi and sequential decoding," IEEE Transactions on Communications, vol. 37 , Issue 11, pp. $1113-1125,1989$.

[12] Dhirendra Kumar Tripathi, S.Arulmozhi Nangai, R. Muthaiah, "FPGA implementation of scalable bandwidth single carrier frequency domain multiple access transceiver for the fourth generation wireless communication," Journal of Theoretical and Applied Information Technology, vol. 28 No.2, June 2011.

[13] H. G. Myung, "Single Carrier Orthogonal Multiple Access Technique for Broadband Wireless Communications," Ph.D. Dissertation, Polytechnic University, January 2007.

[14] W. H. Tranter, K. S. Shanmugan, T. S. Rappaport, and K. L. Kosbar, Principles of Communication Systems Simulation with Wireless Applications, 1st ed., Prentice Hall Professional Technical Reference PTR , 2004..

[15] M. A. Abd El-Hamed, M. I. Dessouky, F. Shawki, Mohammad K. Ibrahim, S. El-Rabaie, and F. E. Abd El-Samie, "Wavelet-Based SCFDMA System," 29th National Radio Science Conference (NRSC 2012), pp. 447 - 460, 2012.

[16] Peng LI, Yu ZHU, Zongxin WANG, and Naibo WANG, "Peak-toaverage power ratio of SC-FDMA systems with localized subcarrier mapping," IEEE Global Mobile Congress GMC2010, pp. 1 - 6, 2010.

[17] Weidong Wang, Yan Zhou, Yuan Sang, Xue Shen, Fan Li, and Yinghai Zhang, "A Ue-interfering area based inter cell interference coordination scheme in SCFDMA uplinks," 2nd IEEE International Conference on Network Infrastructure and Digital Content (IC-NIDC 2010), pp. 681 686, 2010.

[18] F. Classen and H. Meyr, "Frequency synchronization algorithms for OFDM systems suitable for communications over frequency selective fading channels," IEEE 44th Vehicular Technology Conference (VTC), pp. $1655-1659,1994$.

[19] Harri Holma and Antti Toskala, LTE for UMTS: OFDMA and SCFDMA based radio Access, 1st ed., John Wiley \& sons, 2009.

[20] Sosth'ene Yameogo , Jacques Palicot, Laurent Cariou, "Blind time domain equalization of scfdma signal," IEEE 70th Vehicular Technology Conference Fall (VTC2009), pp. 1 - 4, 2009.
[21] 3GPP TS 36.212: Evolved Universal Terrestrial Radio Access (EUTRA); Multiplexing and channel coding, 2009.

[22] Mehmet Kemal Ozdemir, Huseyin Arslan, "Channel estimation for wireless ofdm systems," IEEE Communications Surveys, 2nd Quarter, vol. 9, No. 2, pp. 18-48, 2007.

[23] D. Falconer, S. L. Ariyavisitakul, A. Benyamin-Seeyar, and B. Eidson, "Frequency domain equalization for single-carrier broadband wireless systems," IEEE Communications Magazine, vol. 40 , Issue 4, pp. 58 66, 2002.

[24] Yao Xiao, "Orthogonal frequency division multiplexing modulation and inter-carrier interference cancellation," MSc. Thesis, Louisiana State University, May 2003.

[25] 3GP TS 25.211, 3rd generation partnership project; technical specification group radio access network; Physical Channels and Mapping of Transport Channels onto Physical Channels (FDD), 2009.

[26] John Doble, Introduction to Radio Propagation for Fixed and Mobile Communications, 1 st ed.,Artech House Publishers, 1996.

[27] Bernard Sklar, "Rayleigh fading channels in mobile digital communication systems part i: characterization," IEEE Communications Magazine, vol. 35 , Issue 7, pp. 90 - 100, 1997.

[28] Gordan L. Stuber, Principles of Mobile Communications, 2nd ed., Kluwer Academic Publishers, 2001.

[29] G.J. Foschini and M.J. Gans, "On limits of wireless communications in a fading environment when using multiple antennas," Wireless Personal Communications, vol. 6, pp. 311-335, March 1998.

[30] M. F. Pop and N. C. Beaulieu, "Statistical investigation of sum-ofsinusoids fading channel simulators," Global Telecommunications Conference, (GLOBECOM '99), vol. 1a , pp.419-426, 1999.

[31] S. Rajkumar, "Modelling of multipath fading channels for network simulation," PhD. Dissertation, Texas A\&M University,2007.

[32] Peral Rosado, Lopez Salcedo, Gonzalo Seco , Francesca Zanier, and Massimo Crisci, "Evaluation of the LTE Positioning Capabilities under Typical Multipath Channels," 6th IEEE Advanced Satellite Multimedia Systems Conference ASMS, pp. 139 - 146, 2012.

[33] Ming-Xian Chang; Su, Y.T., "Blind joint channel and data estimation for OFDM signals in Rayleigh fading," IEEE 53rd Vehicular Technology Conference (VTC 2001), vol.2, pp. 791 -795, 2001.

[34] Ye Li, Leonard J. Cimini and Nelson R. Sollenberger, "robust channel estimation for ofdm systems with rapid dispersive fading channels," IEEE International Conference on Communications(ICC 98), pp. 1320 $1324,1998$.

[35] 3G TR 25.943, 3rd Generation Partnership Project; Technical Specification Group (TSG) RAN WG4; Deployment aspects, Stockholm, Sweden, June 2001.

[36] ETSI TR 125943 V4.0.0, Universal Mobile Telecommunications System (UMTS); Deployment aspects, 2006.

[37] Noman Shabbir, Muhammad T. Sadiq, Hasnain Kashif, and Rizwan Ullah, "Comparison of radio propagation models for long term evolution (LTE) network," International Journal of Next-Generation Networks, Vol.3, No.3, 2011.

[38] J. P. Dobbelsteen, "Mapping an LTE Baseband Receiver on a MultiCore Architecture," MSc. Thesis, Eindhoven University Of Technology, 2009.

[39] ITU-R M.2135-1 International Telecommunication Union, Guidelines for evaluation of radio interface technologies for IMT-Advanced, 2008.

[40] Asad Mehmood and Waqas Aslam Cheema, "Channel Estimation For Lte Downlink," MSc. Thesis, Blekinge Institute of Technology, September 2009. 